Article

\title{
Variation of Morphological Traits and Quality Indices of Micropropagated Melia volkensii Gürke Clones before Field Planting
}

\author{
Constantin Dushimimana ${ }^{1,2, *(\mathbb{D}}$, Titus Magomere ${ }^{2}\left(\mathbb{D}\right.$, Jackson Mulatya ${ }^{3}$, Jan Vandenabeele ${ }^{4}$, Florence Olubayo ${ }^{2}$, \\ Guy Smagghe ${ }^{1}\left(\mathbb{D}\right.$ and Stefaan P. O. Werbrouck ${ }^{1}{ }^{\circledR}$
}

Citation: Dushimimana, C.;

Magomere, T.; Mulatya, J.;

Vandenabeele, J.; Olubayo, F.;

Smagghe, G.; Werbrouck, S.P.O

Variation of Morphological Traits and

Quality Indices of Micropropagated

Melia volkensii Gürke Clones before

Field Planting. Forests 2022, 13, 337.

https://doi.org/10.3390/f13020337

Academic Editors: Jorge Canhoto,

Paloma Moncaleán and Sandra

Correia

Received: 25 January 2022

Accepted: 16 February 2022

Published: 18 February 2022

Publisher's Note: MDPI stays neutral with regard to jurisdictional claims in published maps and institutional affiliations.

Copyright: (C) 2022 by the authors. Licensee MDPI, Basel, Switzerland. This article is an open access article distributed under the terms and conditions of the Creative Commons Attribution (CC BY) license (https:// creativecommons.org/licenses/by/ $4.0 /)$.
1 Department of Plants and Crops, Faculty of Bioscience Engineering, Ghent University, Coupure Links 653 and Valentin Vaerwyckweg 1, B-9000 Ghent, Belgium; guy.smagghe@ugent.be (G.S.); stefaan.werbrouck@ugent.be (S.P.O.W.)

2 Department of Plant Science and Crop Protection, University of Nairobi, Kangemi, Nairobi P.O. Box 29053-00625, Kenya; magomere.titus@ku.ac.ke (T.M.); olubayo@uonbi.ac.ke (F.O.)

3 Kenya Forestry Research Institute, Nairobi P.O. Box 20412-00200, Kenya; jmulatya@kefri.org

4 Better Globe Forestry, Nairobi P.O Box 823-00606, Kenya; jan@betterglobeforestry.com

* Correspondence: constantin.dushimimana@ugent.be; Tel.: +254-790-484-424

\begin{abstract}
The quality of acclimatized in vitro cultivated plants is essential to ensure good survival and growth after planting in field conditions. After two months of acclimatization, this study revealed a significant variation in survival rate, shoot characteristics, root traits, and biomass features between 13 clones of Melia volkensii Gürke. A number of quality indices such as the Dickson Quality Index (DQI), shoot dry weight: root dry weight ratio (S:R), and sturdiness quotient (SQ) also showed a large variation. The survival rate was genotype-dependent, with an average of $85 \%$. Extreme genotypes were tall, had long internodes, a lot of leaves, and a large leaf area. At the other side of the spectrum, there were small clones with short internodes and a reduced number of leaves and leaf areas. The high S:R values $(>2)$ of the evaluated clones indicate an imbalance between the shoot and root system, negatively influencing the quality of the in vitro produced $M$. volkensii plants. The SQ for all clones was below the threshold value of 6 , implying a good and expected survival rate. This study illustrates that some clones might systematically score better for $S Q, S: R$, plant volume, and DQI after acclimatization and that these factors could form the basis for selection.
\end{abstract}

Keywords: acclimatization; diversity; in vitro plants; Mukau; roots; morphological

\section{Introduction}

Melia volkensii Gürke (Mukau) is native to the arid and semi-arid savannah of Kenya, Tanzania, and Somalia [1]. The tree grows rapidly and is compatible with agroforestry [2,3]. Mukau leaves, bark, and fruit pulp are fodder [4]. They are also used therapeutically to cure pain and aches [5] and antimicrobial activity [4-7]. Leaf and fruit extracts are used as insect repellants $[8,9]$. The tree is termite resistant $[2,8,10]$ and produces hard timber, comparable to mahogany [11,12]. Pruned branches provide farmers with good firewood [2].

Mukau is primarily propagated by harvesting seeds from selected trees with open pollination, resulting in heterogeneous progeny. The ripening of the fruit from flowering to fruiting takes about 12 to 13 months [4]. Moreover, extracting the tender seeds from the hard fruits is costly [13] and requires challenging skills. Plant tissue culture has the potential to significantly increase the supply of planting material in terms of quality and quantity. Despite previous work on M. volkensii in vitro propagation [14,15], information on the morphological variation between micropropagated clones after acclimatization is still scarce. 
In recent years, our research team has refined the rooting and acclimatization protocols for $M$. volkensii clones [11]. A number of genotypes were selected for their good in vitro propagation, and the plants were successfully acclimatized in the greenhouse. This savannah tree must grow not only fast and reach a good length and stem thickness, but also needs well-developed taproots to withstand dry periods and severe storms. To prevent uprooting, the taproot must also be well oriented. This study aimed to evaluate a number of clones and compare their morphological differences in order to determine whether, after acclimatization, they meet the standards set by farmers for establishment in the field. To this end, a combination of different above and below ground morphological characteristics were recorded and used to determine correlations between them and to calculate tree quality indexes.

\section{Materials and Methods}

\subsection{Initiation and Multiplication}

In this study, thirteen $M$. volkensii clones were used and maintained in vitro. Three clones were initiated from axillary buds of phenotype-selected trees from a progeny field trial conducted by the Kenya Forestry Research Institute (KEFRI) at Tiva (clones 20/21, E34, and E7), Kenya. Shoots of these trees were cut off and surface sterilized by rinsing in ethanol $70 \%$ and incubating in a $20 \% \mathrm{JIK}^{\circledR}$ commercial bleach $(3.5 \% \mathrm{~m} / v$ Sodium hypochlorite) containing $0.005 \%$ detergent (Teepol, Orpington, UK) for $15 \mathrm{~min}$. After rinsing three times in sterile distilled water, meristems were excised under a binocular and transferred to test tubes containing $20 \mathrm{~mL}$ Murashige and Skoog's (MS) medium [16] supplemented with $30 \mathrm{~g} \mathrm{~L}^{-1}$ sucrose, $5 \mu \mathrm{M}$ of meta-Topolin riboside (mTR), and $2 \mathrm{~g} \mathrm{~L}^{-1}$ gelrite.

The remaining clones were derived from mature seeds collected from phenotypically selected, established open-pollinated trees. MAK1 and MAK2 were selected in vitro from seeds harvested from a selected tree in a farmers' orchard in Kibwezi, Kenya. KAS5 (Kasigau origin) was obtained from KEFRI. The remaining clones (19007, 19011, 19016, 19003, 19015, 19008, and K.F1) were obtained from seeds from Better Globe Forestry, Kenya. After removing the pulp, the nut was cracked open, and seeds were selected with an intact seed coat. They were surface sterilized in the same way as the shoots. After the seed coat was cut lengthwise for scarification, they were transferred to test tubes containing MS medium supplemented with $30 \mathrm{~g} \mathrm{~L}^{-1}$ sucrose and $2 \mathrm{~g} \mathrm{~L}^{-1}$ gelrite. The $\mathrm{pH}$ of the medium was adjusted to 5.8 before autoclaving at $121^{\circ} \mathrm{C}$ for $15 \mathrm{~min}$. Two weeks after germination, each seedling was divided into nodes to start micropropagation on the same basal medium supplemented with $5 \mu \mathrm{M}$ mTR. Each subculture lasted four weeks.

\subsection{Rooting}

Four-week-old shoots were cut in explants of approximately $1.5 \mathrm{~cm}$ long with two internodes and leaves. They were rooted using a modified McCown woody plant medium [17] with half concentrations of salts $\left(\mathrm{K}_{2} \mathrm{SO}_{4}\right.$ and $\left.\mathrm{MgSO}_{4}\right)$ and supplemented with $3 \%$ sucrose, $2 \mathrm{~g} \mathrm{~L}^{-1}$ gelrite, $0.02 \mathrm{M}$ Silver Thiosulfate (STS), and $2 \mu \mathrm{M}$ Indole-3-butyric acid (IBA) (pH 5.8 before autoclaving). Per jar of $350 \mathrm{~mL}, 70 \mathrm{~mL}$ autoclaved media was dispersed. The jar had a $0.45 \mu \mathrm{m}$ PTFE filter cup (model TC-GR350, Shanghai Zeshine Equipment Co., Ltd., Shanghai, China). Five explants were transferred into each jar, and cultures were incubated in a growth chamber at $22 \pm 1{ }^{\circ} \mathrm{C}$ with a photoperiod of $16 / 8 \mathrm{~h}$ light/dark. A completely randomized design was used.

\subsection{Acclimatization and Experimental Design}

The rooted in vitro shoots were manually stripped of the remaining adherent medium and washed with tap water. Then, the plantlets were treated with $0.2 \%$ Pearl ${ }^{\circledR} 50$ SC (Osho Chemical Industries Ltd., Nairobi, Kenya) and planted in $300 \mathrm{~mL}$ pots with peat moss (Kekkilä LSM 2w R8264). The pots were covered with clear plastic cups for 12 days (Figure 1a). The pots were watered three times a week. Through irrigation, fertilization with Rosasol NPK 19.19.19+ TE (Rosasol-Even, Elgon Kenya Ltd., Nairobi, Kenya) was 
applied at two-week intervals. The experiments were carried out in a greenhouse located at Kabete field station. The area is situated on $1^{\circ} 14^{\prime} 52^{\prime \prime} \mathrm{S}$ latitude and $36^{\circ} 44^{\prime} 32^{\prime \prime} \mathrm{E}$ longitude, at an altitude of $1846 \mathrm{~m}$ above sea level. The average temperature of the greenhouse was $27^{\circ} \mathrm{C}$.

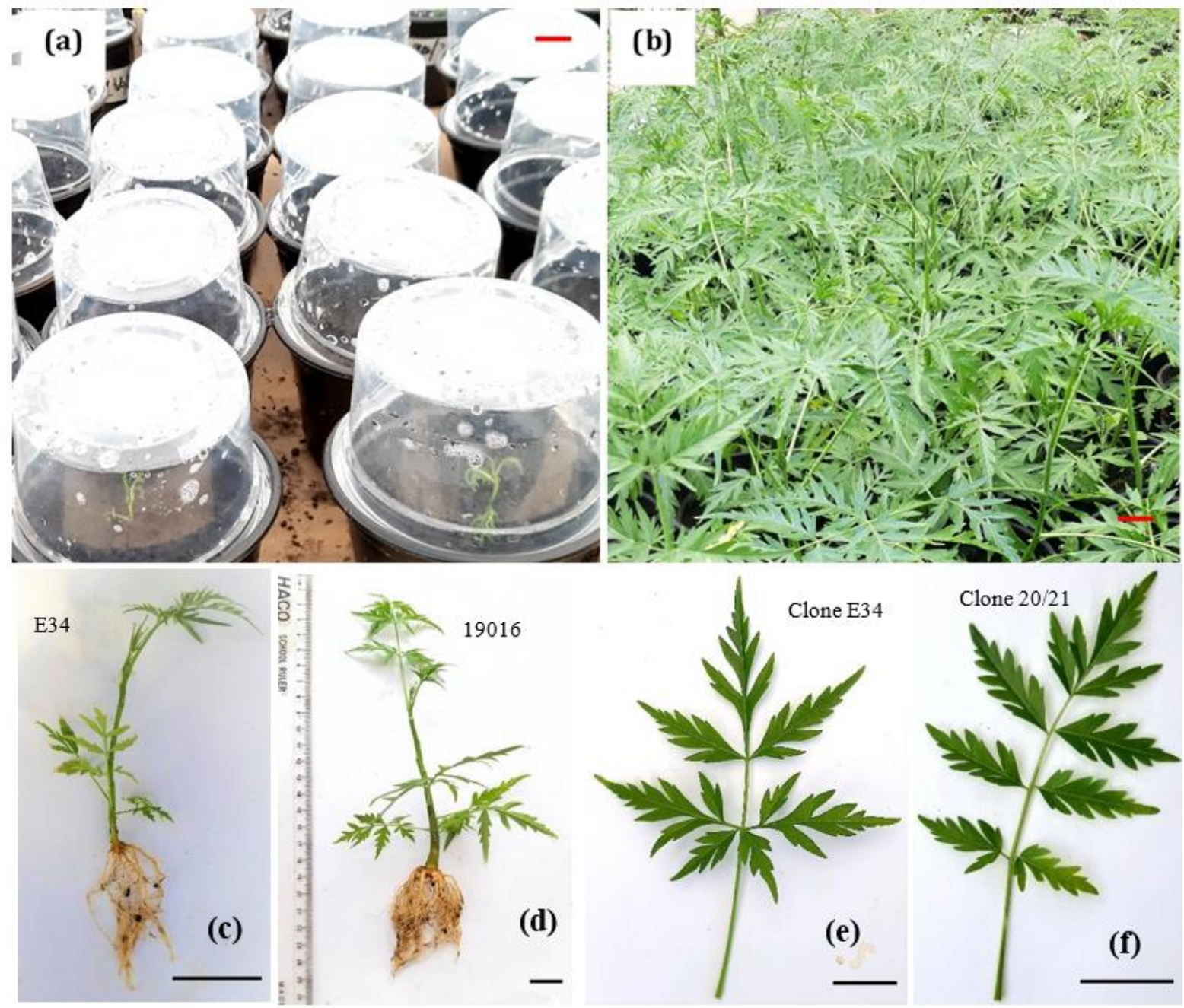

Figure 1. Acclimatization of in vitro propagated M. volkensii Gürke clones. (a) Covered plantlets with clear pots after planting. (b-d) Fully acclimatized M. volkensii plantlets. (e,f) Two different leaf morphology of M. volkensii; (e) leaf with five leaflets and (f) leaf with nine leaflets. (Scale bar $=1 \mathrm{~cm}$ ).

\subsection{Data Collection}

Data were collected two months after transplanting. Parameters recorded were survival rate $(\%)$, plant height $(\mathrm{PH})$ in $\mathrm{cm}$, number of leaves per plant (NL), number of leaflets per leaf (NLL), internode length (INL) in $\mathrm{mm}$, chlorophyll (SPAD value) (CHL), stem diameter (SD) in $\mathrm{mm}$, leaf area (LA) in $\mathrm{cm}^{2}$, and shoot fresh weight (SFW) and dry weight (SDW) in grams. Stem diameter was measured at the first leaf using a digital vernier caliper. The mean leaf area was determined from the top five leaves of each plant through ImageJ (https: / /imagej.nih.gov/ij/, accessed on 15 May 2021). Chlorophyll content was determined using a SPAD meter (Konica Minolta SPAD-502Plus, Sakai, Osaka, Japan). Root traits (number of roots per plant, root diameter, root length $(\mathrm{cm})$, collar diameter (CD) in $\mathrm{mm}$, root fresh (RFW) and dry weight (RDW) in grams, and leaf area were collected from 10 plants per clone from each repetition, except for the one with low survival rate. Shoot and roots were oven-dried at $70^{\circ} \mathrm{C}$ for $72 \mathrm{~h}$ before the shoot and roots dry weight determination. The shoot to root dry mass ratio (S:R) is an essential measure of seedling survival. It relates the evaporating surface to the water-absorbing surface. A healthy plant has a S:R between 1:1 and 1:2 [18,19]. The sturdiness quotient (SQ) was calculated as the 
ratio of plant height $(\mathrm{cm})$ to collar diameter $(\mathrm{mm})$. The smaller the SQ value, the more robust the plant and the higher the expected survival rate, especially in windy or dry places. An SQ higher than 6 is undesirable [18]. The Dickson's Quality Index (DQI) reflects the plants' ability to survive and grow in the field, with performance increasing as the index values rise. It was calculated according to the following Formula (1) [20]:

$$
\mathrm{DQI}=\frac{\text { Plant Dry weight }(\mathrm{g})}{\frac{\text { Height }(\mathrm{cm})}{\text { Collar diameter }(\mathrm{mm})}+\frac{\text { Shoot dry weight }(\mathrm{g})}{\text { Root dry weight }(\mathrm{g})}}
$$

Plants with an index greater than or equal to 0.2 [21] are considered to be of good quality. The aerial plant volume (V) was calculated using the following Formula (2):

$$
\mathrm{V}=\frac{1}{3} \times \pi \times\left(\frac{\mathrm{CD}}{2}\right)^{2} \times \mathrm{H}
$$

where

$\mathrm{V}=$ Aerial plant volume;

$\mathrm{CD}=$ Collar diameter $(\mathrm{mm})$;

$\mathrm{H}=$ Plant height $(\mathrm{cm})$.

\subsection{Statistical Analysis}

Each treatment consisted of at least 30 plants per clone, and this was done in a completely randomized design (CRD). The experiment was repeated three times so that a total of 90 plants were used per clone. ANOVA was performed using IBM $^{\circledR}$ SPSS ${ }^{\circledR}$ statistics (version 28). A post-hoc Duncan's multiple range test $(p<0.05)$ was performed to separate the means.

\section{Results}

\subsection{Shoot Morphological Traits}

The plantlets were successfully acclimatized (Figure $1 \mathrm{~b}-\mathrm{d}$ ) with a mean survival rate of $85.4 \%$. There was a highly significant $(p<0.001)$ difference in survival rate between the clones. Clone 19016 had the highest survival rate, while clone 19003 had the lowest. There were highly significant $(p<0.001)$ differences for all shoot traits among elite $M$. volkensii clones after two months of acclimatization (Table 1). Clone plant height varied from $5.94 \mathrm{~cm}$ for 19003 to $15.81 \mathrm{~cm}$ for 19008. The lowest average number of leaves per plant (7.46) was recorded in clone 19003, while the highest number of leaves per plant (11.68) was recorded in clone 19016. The number of leaflets per leaf ranged from five (Figure 1e) to seven (Figure 1f). Three clones, namely 20/21, E7, and K.F1, had the highest number of leaflets per leaf, namely seven. The lowest number of leaflets per plant of 5.30, 5.64, and 5.64 were recorded from clone E34, 19008, and 19003, respectively. Internode length ranged from $1.44 \mathrm{~mm}$ for clone 19003 to $2.96 \mathrm{~mm}$ for clone 19008. Clone 19008 had significantly the highest mean internode length. Chlorophyll content (SPAD meter value) varied from 23.91 for KAS5 to 30.07 for 19003 . There was no significant difference between the chlorophyll content of clone 19003 and the other eleven clones, namely K.F1, E7, MAK1, MAK2, 19016, 19015, 19008, E34, 19007, 20/21, and 19011. The thickest stems, 3.6 and $3.4 \mathrm{~mm}$, were recorded with MAK1 and 19007, respectively, while 20/21 and 9015 had the smallest stem diameters, measuring at 2.3 and $2.4 \mathrm{~mm}$. Leaf area ranged from $16.99 \mathrm{~cm}^{2}$ for 19003 to $41.87 \mathrm{~cm}^{2}$ for 19016. Seven clones, namely 19003, 20/21, E34, MAK2, KAS5, E7, and 19011, had significantly smaller leaf areas than the other clones. Shoot fresh weight ranged from $1.95 \mathrm{~g}$ to $5.67 \mathrm{~g}$ for all clones. The SFW of clones 19016 and MAK1 was similar. The lightest shoots were recorded in clones 19003, 20/21, E34, KAS5, and E7 with 1.95, 2.09, 2.55, 2.99, and $3.01 \mathrm{~g}$, respectively. Clone coded 19016 and MAK1 had the best SDW (1.14 and $1.05 \mathrm{~g})$, while the lowest SDW was recorded for 20/21, 19003, and E34 with mean values of 0.32, 0.38 , and $0.43 \mathrm{~g}$, respectively. 
Table 1. Mean values of survival rate and shoot traits variation among 13 elite Melia volkensii Gürke clones after two months of acclimatization.

\begin{tabular}{|c|c|c|c|c|c|c|}
\hline Clones Code & Survival Rate & PH (cm) & NL & NLL & INL (mm) & CHL \\
\hline 19015 & $95.0 \pm 0.64^{\mathrm{a}}$ & $10.5 \pm 0.55^{\text {cde }}$ & $9.8 \pm 0.18^{b}$ & $5.9 \pm 0.12$ ef & $1.9 \pm 0.09^{\mathrm{d}}$ & $28.4 \pm 0.71^{\mathrm{a}}$ \\
\hline 19011 & $93.3 \pm 6.67^{\mathrm{a}}$ & $12.2 \pm 0.60 \mathrm{bc}$ & $10.3 \pm 0.29 b c$ & $6.4 \pm 0.13^{\text {cde }}$ & $2.5 \pm 0.15^{\mathrm{abc}}$ & $26.2 \pm 0.73^{\mathrm{ab}}$ \\
\hline $20 / 21$ & $84.0 \pm 8.74^{\mathrm{a}}$ & $9.1 \pm 0.42 \mathrm{de}$ & $8.2 \pm 0.18^{\mathrm{f}}$ & $7.2 \pm 0.14^{\mathrm{a}}$ & $2.1 \pm 0.10^{\mathrm{cd}}$ & $26.4 \pm 2.25^{\mathrm{ab}}$ \\
\hline K.F1 & $95.9 \pm 2.41^{\mathrm{a}}$ & $10.6 \pm 0.67^{\mathrm{cd}}$ & $9.9 \pm 0.23 b c$ & $7.0 \pm 0.17^{a b}$ & $2.0 \pm 0.19 \mathrm{bcd}$ & $30.0 \pm 0.99^{a}$ \\
\hline KAS5 & $98.0 \pm 1.96^{\mathrm{a}}$ & $9.1 \pm 0.55^{\mathrm{de}}$ & $9.6 \pm 0.26^{\mathrm{cd}}$ & $6.48 \pm 0.16^{b c d}$ & $1.9 \pm 0.13^{\mathrm{de}}$ & $23.9 \pm 0.71^{b}$ \\
\hline MAK1 & $97.6 \pm 1.19^{\mathrm{a}}$ & $12.0 \pm 0.55^{b c}$ & $9.7 \pm 0.20 \mathrm{bcd}$ & $6.8 \pm 0.09 \mathrm{abc}$ & $2.2 \pm 0.11^{\mathrm{bcd}}$ & $29.1 \pm 0.67^{\mathrm{a}}$ \\
\hline 19008 & $83.8 \pm 10.11^{\mathrm{a}}$ & $15.8 \pm 0.85^{\mathrm{a}}$ & $9.8 \pm 0.29 \mathrm{bcd}$ & $5.6 \pm 0.18^{\mathrm{fg}}$ & $3.0 \pm 0.18^{a}$ & $27.9 \pm 0.96^{\mathrm{a}}$ \\
\hline E34 & $58.0 \pm 20.44^{b}$ & $8.4 \pm 0.82^{\mathrm{e}}$ & $9.0 \pm 0.32 \mathrm{de}$ & $5.3 \pm 0.13^{g}$ & $2.1 \pm 0.24^{\mathrm{bcd}}$ & $27.0 \pm 0.87^{a b}$ \\
\hline MAK2 & $95.9 \pm 3.23^{a}$ & $12.4 \pm 0.87^{b c}$ & $9.8 \pm 0.23 \mathrm{bcd}$ & $6.5 \pm 0.14^{\mathrm{bcd}}$ & $2.6 \pm 0.22 \mathrm{ab}$ & $28.9 \pm 0.83^{a}$ \\
\hline 19003 & $34.0 \pm 9.52^{c}$ & $5.9 \pm 0.69^{\mathrm{f}}$ & $7.5 \pm 0.51^{\mathrm{g}}$ & $5.6 \pm 0.24 \mathrm{fg}$ & $1.4 \pm 0.19^{\mathrm{e}}$ & $30.1 \pm 1.14^{\mathrm{a}}$ \\
\hline 19007 & $85.9 \pm 6.49^{a}$ & $13.6 \pm 0.89^{b}$ & $10.5 \pm 0.29^{b}$ & $6.2 \pm 0.16^{\mathrm{de}}$ & $2.3 \pm 0.17^{\mathrm{bcd}}$ & $26.6 \pm 0.67^{\mathrm{ab}}$ \\
\hline 19016 & $99.0 \pm 1.01^{\mathrm{a}}$ & $14.1 \pm 0.53^{\mathrm{ab}}$ & $11.7 \pm 0.16^{\mathrm{a}}$ & $6.5 \pm 0.09 \mathrm{~cd}$ & $2.6 \pm 0.12^{a b c}$ & $28.6 \pm 0.53^{a}$ \\
\hline E7 & $91.7 \pm 4.79^{\mathrm{a}}$ & $10.7 \pm 0.66^{\mathrm{cd}}$ & $8.6 \pm 0.25^{\text {ef }}$ & $7.0 \pm 0.18^{a}$ & $2.3 \pm 0.16^{\mathrm{bcd}}$ & $29.2 \pm 0.77^{\mathrm{a}}$ \\
\hline Mean & 85.4 & 11.36 & 9.72 & 6.48 & 2.24 & 27.84 \\
\hline LSD 0.05 & 23.1 & 2.42 & 0.90 & 0.54 & 0.57 & 4.04 \\
\hline Clones Code & \multicolumn{2}{|c|}{$\mathrm{SD}(\mathrm{mm})$} & LA $\left(\mathrm{cm}^{2}\right)$ & SFW (g) & \multicolumn{2}{|c|}{ SDW (g) } \\
\hline 19015 & \multicolumn{2}{|c|}{$2.4 \pm 0.05 \mathrm{gh}$} & $29.8 \pm 2.73 \mathrm{bcd}$ & $3.3 \pm 0.25^{\text {cde }}$ & \multicolumn{2}{|c|}{$0.6 \pm 0.05^{\text {def }}$} \\
\hline 19011 & \multicolumn{2}{|c|}{$2.9 \pm 0.06^{\mathrm{def}}$} & $25.9 \pm 2.56^{\text {bcde }}$ & $3.8 \pm 0.38^{\mathrm{cd}}$ & \multicolumn{2}{|c|}{$0.6 \pm 0.06^{\mathrm{de}}$} \\
\hline $20 / 21$ & \multicolumn{2}{|c|}{$2.3 \pm 0.04^{h}$} & $19.3 \pm 1.47^{\mathrm{e}}$ & $2.1 \pm 0.19^{f}$ & \multicolumn{2}{|c|}{$0.3 \pm 0.03 \mathrm{~g}$} \\
\hline K.F1 & \multicolumn{2}{|c|}{$3.3 \pm 0.06^{b c}$} & $32.9 \pm 2.93^{b}$ & $4.1 \pm 0.36^{\mathrm{bcd}}$ & \multicolumn{2}{|c|}{$0.7 \pm 0.07^{\mathrm{cd}}$} \\
\hline KAS5 & \multicolumn{2}{|c|}{$3.1 \pm 0.08^{\mathrm{cd}}$} & $24.8 \pm 2.53$ bcde & $3.0 \pm 0.31$ def & \multicolumn{2}{|c|}{$0.6 \pm 0.06$ def } \\
\hline MAK1 & \multicolumn{2}{|c|}{$3.6 \pm 0.08^{a}$} & $31.2 \pm 2.48 \mathrm{bc}$ & $5.0 \pm 0.35^{\mathrm{ab}}$ & \multicolumn{2}{|c|}{$1.0 \pm 0.08^{a b}$} \\
\hline 19008 & \multicolumn{2}{|c|}{$2.9 \pm 0.09 \mathrm{def}$} & $29.0 \pm 3.04 \mathrm{bcd}$ & $3.8 \pm 0.37^{\mathrm{cd}}$ & \multicolumn{2}{|c|}{$0.9 \pm 0.08^{b c}$} \\
\hline E34 & \multicolumn{2}{|c|}{$2.6 \pm 0.08^{\mathrm{fg}}$} & $20.7 \pm 3.55^{\mathrm{de}}$ & $2.5 \pm 0.40^{\text {ef }}$ & \multicolumn{2}{|c|}{$0.4 \pm 0.07^{\text {efg }}$} \\
\hline MAK2 & \multicolumn{2}{|c|}{$3.3 \pm 0.10^{b c}$} & $23.0 \pm 2.51 \mathrm{cde}$ & $3.3 \pm 0.31^{\mathrm{cde}}$ & \multicolumn{2}{|c|}{$0.6 \pm 0.05^{\mathrm{de}}$} \\
\hline 19003 & \multicolumn{2}{|c|}{$2.8 \pm 0.16^{\text {ef }}$} & $17.0 \pm 2.59 \mathrm{e}$ & $1.9 \pm 0.32^{\mathrm{f}}$ & \multicolumn{2}{|c|}{$0.4 \pm 0.09 \mathrm{fg}$} \\
\hline 19007 & \multicolumn{2}{|c|}{$3.4 \pm 0.10^{a b}$} & $31.4 \pm 3.69 \mathrm{bc}$ & $4.3 \pm 0.51 \mathrm{bc}$ & \multicolumn{2}{|c|}{$1.0 \pm 0.13^{\mathrm{ab}}$} \\
\hline 19016 & \multicolumn{2}{|c|}{$3.3 \pm 0.10^{b c}$} & $41.9 \pm 2.74^{\mathrm{a}}$ & $5.7 \pm 0.30^{\mathrm{a}}$ & \multicolumn{2}{|c|}{$1.1 \pm 0.06^{\mathrm{a}}$} \\
\hline E7 & \multicolumn{2}{|c|}{$2.9 \pm 0.08^{\text {de }}$} & $25.5 \pm 2.68$ bcde & $3.0 \pm 0.28$ def & \multicolumn{2}{|c|}{$0.6 \pm 0.05$ de } \\
\hline Mean & \multicolumn{2}{|c|}{3.01} & 27.74 & 3.62 & \multicolumn{2}{|c|}{0.71} \\
\hline LSD 0.05 & & & 9.87 & 1.22 & & \\
\hline
\end{tabular}

PH: plant height $(\mathrm{cm})$. NL: number of leaves per plant. NLL: number of leaflets per leaf. INL: internode length $(\mathrm{mm})$. CHL: chlorophyll content (SPAD meter). SD: stem diameter in $\mathrm{mm}$. LA: leaf area $\left(\mathrm{cm}^{2}\right)$. SFW: shoot fresh weight. SDW: shoot dry weight. LSD: least significant differences of means at $5 \%$. Means followed by the same letters within a column are not significantly different at $p \leq 0.05$ (Duncan's multiple range test at $5 \%$ ). Data are presented as mean \pm standard errors. $N=90$ except for SFW and SDW, where $N=30$.

\subsection{Root Morphology}

The mean values for the number of roots per plant, root length, root diameter, and collar diameter are presented in Table 2. Significant $(p<0.001)$ variations were observed in root traits among clones (Figure 2). The highest number of roots per plant were recorded from clone 19016, followed by 20/21, K.F1, 19015, and 19007. Eight clones, namely 19008, E34, E7, 19003, MAK3, MAK1, 19011, and KAS5, recorded the lowest number of roots per plant after two months under acclimatization. Clone 19011 had the longest roots $(11.6 \mathrm{~cm})$ and E34 the shortest. The largest root diameter and collar diameter were measured at MAK1 (7.7 mm). Clones such as 20/21, E34, 19003, and 19015 developed the smallest root diameters with mean values of $1.9,2.1,2.5$, and $2.5 \mathrm{~mm}$, respectively. The smallest collar diameters (CD) were recorded as 4.2, 4.3, 4.3, and $4.9 \mathrm{~mm}$, respectively, from clones 19015, 20/21, E34, and 19003. The roots of MAK1 were significantly heavier compared to other clones. Contrastingly, clone 20/21 was recorded as possessing the lowest RFW with a mean value of $1.0 \mathrm{~g}$. The RDW varied between $0.1 \mathrm{~g}$ to $0.5 \mathrm{~g}$ for all clones. The heaviest RDW was recorded for MAK1, while the lightest were recorded for 20/21, E34, 19003, and 19015. 
Table 2. Number of roots per plant, root length, root diameter, collar diameter, and root biomass of elite Melia volkensii clones seedling after two months under acclimatization.

\begin{tabular}{|c|c|c|c|c|c|c|}
\hline Clones Code & NR & RL (cm) & $\mathrm{RD}(\mathrm{mm})$ & $\mathrm{CD}(\mathrm{mm})$ & RFW (g) & RDW (g) \\
\hline 19015 & $6.8 \pm 0.66^{\mathrm{abc}}$ & $10.8 \pm 0.48^{\mathrm{abc}}$ & $2.5 \pm 0.14^{\mathrm{d}}$ & $4.2 \pm 0.13^{\mathrm{e}}$ & $1.0 \pm 0.08^{\mathrm{e}}$ & $0.1 \pm 0.01^{\mathrm{de}}$ \\
\hline 19011 & $4.1 \pm 0.42^{\mathrm{e}}$ & $11.6 \pm 0.50^{\mathrm{a}}$ & $4.8 \pm 0.31^{b}$ & $6.3 \pm 0.31^{b}$ & $1.9 \pm 0.16^{b c}$ & $0.2 \pm 0.02^{b}$ \\
\hline $20 / 21$ & $7.5 \pm 0.60^{\mathrm{ab}}$ & $9.4 \pm 0.32$ ef & $1.9 \pm 0.11^{\mathrm{d}}$ & $4.3 \pm 0.17^{\mathrm{e}}$ & $0.5 \pm 0.04^{\mathrm{f}}$ & $0.1 \pm 0.01^{\mathrm{e}}$ \\
\hline K.F1 & $7.2 \pm 0.72^{a b}$ & $10.3 \pm 0.48^{b c d}$ & $4.2 \pm 0.19 b c$ & $6.4 \pm 0.22^{b}$ & $1.7 \pm 0.11^{\mathrm{cd}}$ & $0.2 \pm 0.01 \mathrm{bc}$ \\
\hline KAS5 & $4.1 \pm 0.47^{\mathrm{e}}$ & $10.0 \pm 0.46^{\text {bcde }}$ & $4.7 \pm 0.26^{b}$ & $5.6 \pm 0.16^{b c}$ & $1.5 \pm 0.15^{\mathrm{d}}$ & $0.2 \pm 0.01^{b c}$ \\
\hline MAK1 & $4.7 \pm 0.55^{\mathrm{de}}$ & $11.1 \pm 0.37^{\mathrm{ab}}$ & $7.7 \pm 0.43^{a}$ & $7.7 \pm 0.43^{a}$ & $3.8 \pm 0.17^{\mathrm{a}}$ & $0.5 \pm 0.03^{a}$ \\
\hline 19008 & $5.9 \pm 0.74$ bcde & $8.6 \pm 0.20^{g}$ & $3.7 \pm 0.19^{c}$ & $5.1 \pm 0.17^{\mathrm{cd}}$ & $1.4 \pm 0.10^{\mathrm{d}}$ & $0.1 \pm 0.01^{\mathrm{cd}}$ \\
\hline E34 & $5.8 \pm 0.64$ bcde & $8.1 \pm 0.32^{g}$ & $2.1 \pm 0.15^{\mathrm{d}}$ & $4.3 \pm 0.19^{\mathrm{e}}$ & $1.0 \pm 0.12^{\mathrm{e}}$ & $0.2 \pm 0.01^{\mathrm{e}}$ \\
\hline MAK2 & $5.0 \pm 0.55^{\text {cde }}$ & $9.8 \pm 0.31^{\mathrm{cdef}}$ & $4.0 \pm 0.29 b c$ & $6.1 \pm 0.33^{b}$ & $1.6 \pm 0.09 \mathrm{~cd}$ & $0.2 \pm 0.01^{b c}$ \\
\hline 19003 & $5.0 \pm 0.81^{\mathrm{cde}}$ & $9.0 \pm 0.70^{\text {efg }}$ & $2.5 \pm 0.27^{\mathrm{d}}$ & $4.9 \pm 0.28 \mathrm{de}$ & $1.0 \pm 0.17^{\mathrm{e}}$ & $0.1 \pm 0.01^{\mathrm{e}}$ \\
\hline 19007 & $6.6 \pm 0.75^{\mathrm{abcd}}$ & $9.8 \pm 0.34$ def & $4.2 \pm 0.23^{b c}$ & $6.0 \pm 0.19^{b}$ & $2.0 \pm 0.17^{b c}$ & $0.2 \pm 0.02^{b}$ \\
\hline 19016 & $8.4 \pm 0.66^{a}$ & $8.7 \pm 0.21$ efg & $4.4 \pm 0.27^{b c}$ & $5.7 \pm 0.16^{b c}$ & $2.2 \pm 0.09^{b}$ & $0.2 \pm 0.01^{b}$ \\
\hline E7 & $5.5 \pm 0.55^{\text {bcde }}$ & $10.0 \pm 0.44^{\text {bcde }}$ & $3.6 \pm 0.23^{c}$ & $5.8 \pm 0.21 \mathrm{bc}$ & $1.5 \pm 0.11^{\mathrm{d}}$ & $0.2 \pm 0.01^{b c d}$ \\
\hline Mean & 5.95 & 9.87 & 3.98 & 5.62 & 1.67 & 0.18 \\
\hline LSD 0.05 & 2.20 & 1.41 & 0.90 & 0.87 & 0.44 & 0.06 \\
\hline
\end{tabular}

NR: number of roots per plant. RL: root length $(\mathrm{cm})$. RD: root diameter. CD: collar diameter (mm). RFW: root fresh weight. RDW: root dry weight. LSD: least significant differences of means at $5 \%$. Means followed by the same letters within a column are not significantly different at $p \leq 0.05$ (Duncan's multiple range test at $5 \%$ ). Data are presented as mean \pm standard errors. $N=30$.
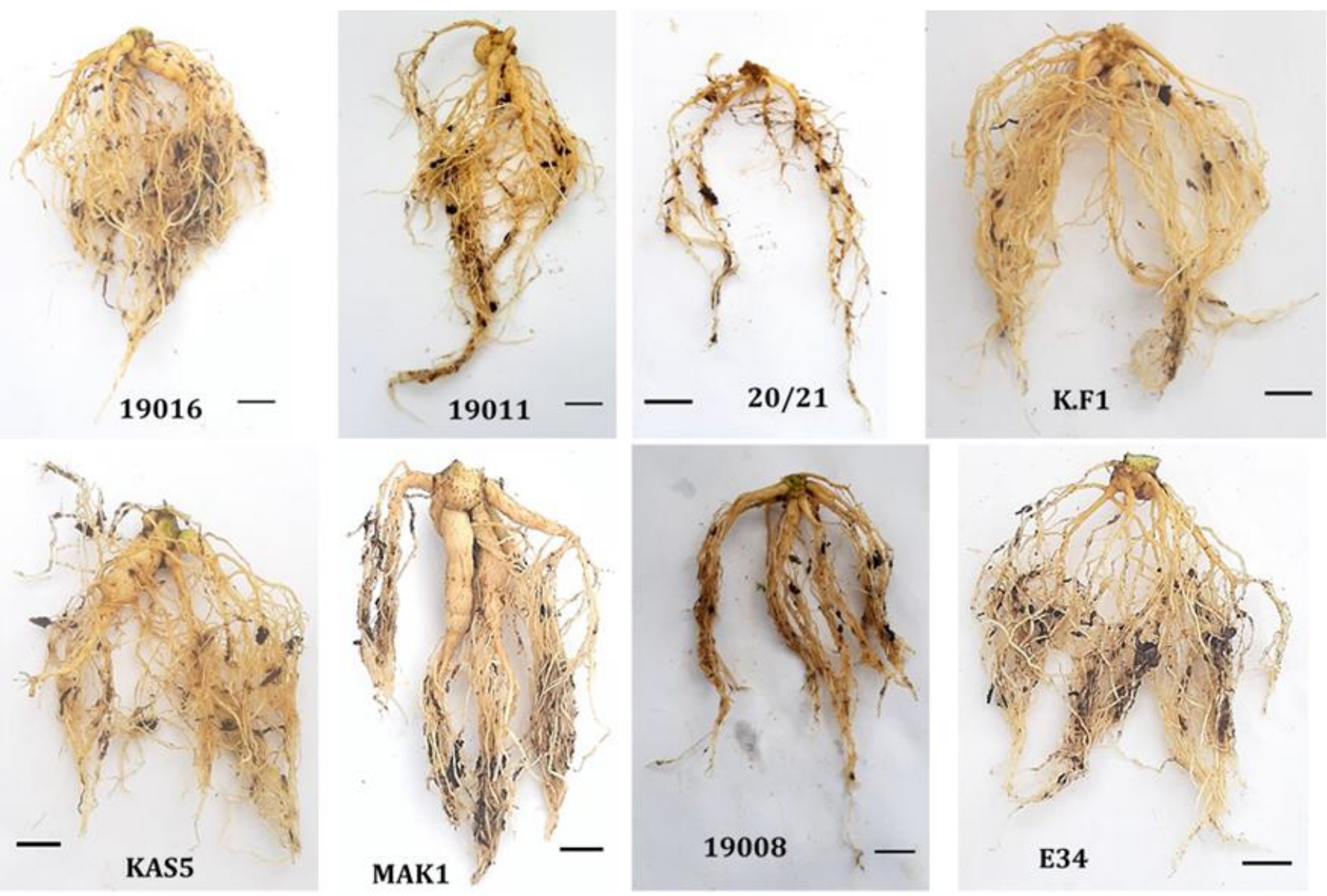

Figure 2. Cont. 

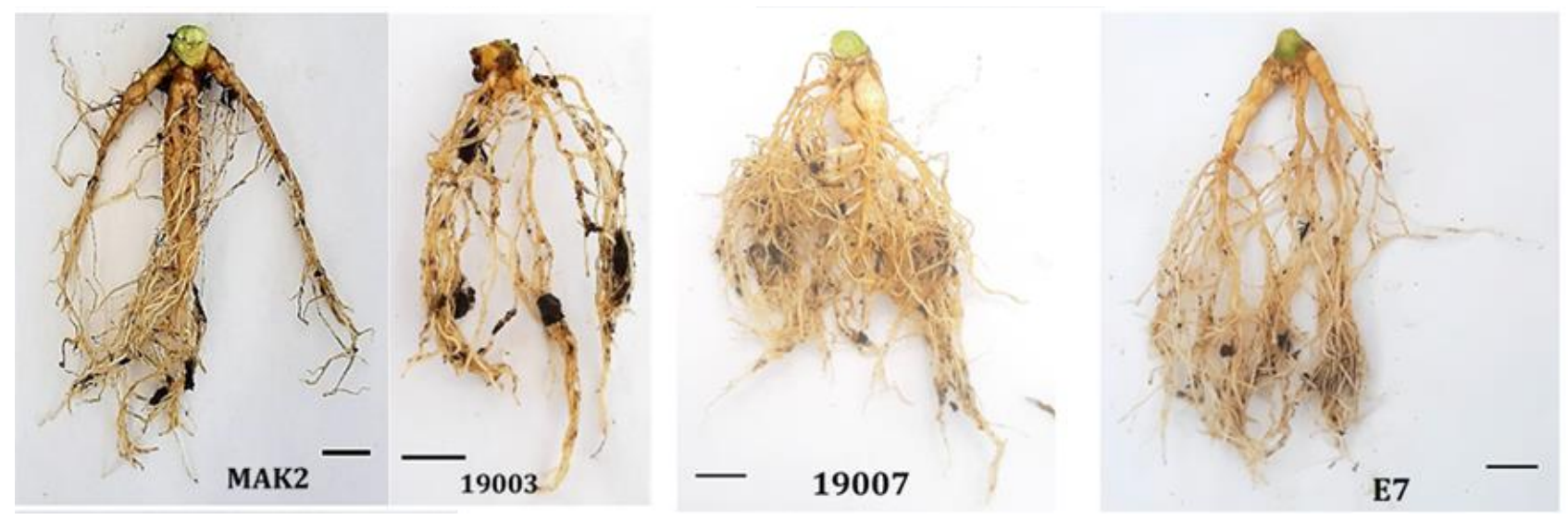

Figure 2. Morphological diversity of the root system of $M$. volkensii clones after two months of acclimatization under greenhouse conditions (scale bar $=1 \mathrm{~cm}$ ).

\subsection{Quality Indexes}

There were highly significant $(p<0.001)$ differences for SQ, DQI, and S:R among M. volkensii clones (Table 3). Sturdiness quotient ranged from 1.33 for clone 19003 to 2.74 for 19008. All clones had an SQ below the threshold of 6, which is recommended for robust young trees. The highest S:R ratio values of 7.58 were observed for 19015, while the lowest values of 2.35 and 3.45 were recorded for MAK1 and 19011. Since almost all evaluated clones had an S:R higher than the recommended value $(\leq 1)$, even MAK1, it can be concluded that all acclimatized plants developed a disproportionately light root system in the pots. DQI index varied significantly among clones. The DQI ranged between 0.05 for $20 / 21$ and 0.48 for MAK1. Seven clones out of thirteen had the average recommended value of $\geq 0.2$. The plant volume significantly varied among clones. Giant plants were observed in clones MAK1 with a mean shoot volume of 171.3, followed by clone 19007 (120.2), 19016 (119.3), K.F1 (113.1), and 19011, respectively. The plants with smaller volumes were recorded from 20/21 (41.6), 19003 (41.9), 19015 (47.7), and E34 (42.8), respectively. Clone ranking using plant volume index was comparable with the Dickson quality index ranking.

Table 3. Variation in quality indexes of 13 selected Melia volkensii clones after acclimatization.

\begin{tabular}{ccccc}
\hline Clones Code & DQI & SQ & S:R & V \\
\hline 19015 & $0.07 \pm 0.006^{\mathrm{de}}$ & $2.50 \pm 0.124^{\mathrm{abc}}$ & $7.58 \pm 0.600^{\mathrm{a}}$ & $47.7 \pm 3.68^{\mathrm{d}}$ \\
19011 & $0.18 \pm 0.016^{\mathrm{b}}$ & $1.97 \pm 0.168^{\mathrm{cd}}$ & $3.45 \pm 0.291^{\text {ef }}$ & $108.8 \pm 8.36^{\mathrm{b}}$ \\
$20 / 21$ & $0.05 \pm 0.006^{\mathrm{e}}$ & $2.14 \pm 0.156^{\mathrm{bcd}}$ & $6.15 \pm 0.365^{\mathrm{b}}$ & $41.6 \pm 3.34^{\mathrm{d}}$ \\
K.F1 & $0.16 \pm 0.010^{\mathrm{bc}}$ & $1.90 \pm 0.188^{\mathrm{cde}}$ & $4.48 \pm 0.567^{\mathrm{cde}}$ & $113.1 \pm 9.68^{\mathrm{b}}$ \\
KAS5 & $0.17 \pm 0.020^{\mathrm{bc}}$ & $1.72 \pm 0.168^{\mathrm{de}}$ & $3.82 \pm 0.622^{\mathrm{e}}$ & $73.9 \pm 6.59^{\mathrm{c}}$ \\
MAK1 & $0.48 \pm 0.051^{\mathrm{a}}$ & $1.75 \pm 0.160^{\mathrm{de}}$ & $2.35 \pm 0.234^{\mathrm{f}}$ & $171.3 \pm 13.43^{\mathrm{a}}$ \\
19008 & $0.12 \pm 0.009^{\mathrm{cd}}$ & $2.74 \pm 0.219^{\mathrm{a}}$ & $6.14 \pm 0.457^{\mathrm{b}}$ & $95.3 \pm 8.31^{\mathrm{bc}}$ \\
E34 & $0.06 \pm 0.008^{\mathrm{de}}$ & $2.09 \pm 0.222^{\mathrm{bcd}}$ & $5.73 \pm 0.401^{\mathrm{bc}}$ & $42.8 \pm 5.91^{\mathrm{d}}$ \\
MAK2 & $0.16 \pm 0.016^{\mathrm{bc}}$ & $1.95 \pm 1.218^{\mathrm{cd}}$ & $4.10 \pm 0.380^{\mathrm{de}}$ & $96.1 \pm 7.91^{\mathrm{bc}}$ \\
19003 & $0.07 \pm 0.013^{\mathrm{de}}$ & $1.33 \pm 0.145^{\mathrm{e}}$ & $5.57 \pm 0.975^{\mathrm{bcd}}$ & $41.9 \pm 6.35^{\mathrm{d}}$ \\
19007 & $0.17 \pm 0.014^{\mathrm{bc}}$ & $2.27 \pm 0.239^{\mathrm{abcd}}$ & $4.71 \pm 0.378^{\mathrm{bcde}}$ & $120.2 \pm 10.80^{\mathrm{b}}$ \\
19016 & $0.17 \pm 0.011^{\mathrm{bc}}$ & $2.59 \pm 0.161^{\mathrm{ab}}$ & $5.76 \pm 0.394^{\mathrm{bc}}$ & $119.3 \pm 7.90^{\mathrm{b}}$ \\
E7 & $0.14 \pm 0.011^{\mathrm{bc}}$ & $1.80 \pm 0.193^{\mathrm{de}}$ & $4.41 \pm 0.405^{\mathrm{cde}}$ & $76.3 \pm 4.70^{\mathrm{c}}$ \\
Mean & 0.16 & 2.08 & 4.88 & $91.6^{6}$ \\
LSD 0.05 & 0.07 & 0.66 & 1.62 & 29.14 \\
\hline
\end{tabular}

SQ: sturdiness quotient (plant height to collar diameter ratio) (value less than 6 indicates good quality plantlets [18] $\mathrm{S}: \mathrm{R}$ : shoot to root ratio (S:R $\leq 1$ indicates good quality plantlets [19]. DQI: Dickson quality index (DQI $\geq 0.2$ denotes good quality plants). V: plant volume. LSD: least significant differences of means at $5 \%$. Means followed by the same letters within a column are not significantly different at $p \leq 0.05$ (Duncan's multiple range test at 5\%). Data are presented as mean \pm standard errors. $N=30$. 


\subsection{Correlation between Morphological Characteristics and Quality of In Vitro Raised Melia volkensii Plants}

A strong correlation was recorded between several shoot and root parameters and quality indexes (Table 4). Plant height was strongly correlated ( $r=0.7-1)$ with INL and SDW and moderately associated with LA, SQ, and SFW. The number of leaves per plant significantly correlated with LA, SFW, and SDW. Stem diameter was strongly correlated with RD, CD, RFW, and RDW. Average leaf area strongly correlated with the NL, SFW, and SDW. Root diameter strongly correlated with CD, RFW, and RDW. It was clear that shoot and root's fresh and dry weight were significantly correlated. As a total dry weight, CD and RDW are positive elements of the DQI; they were obviously highly associated with it. However, SDW, a negative component of DQI, is still positively correlated with it. DQI was moderately correlated $(r=0.5-0.7)$ with RL and SFW. Plant volume was strongly associated with SD, RD, DQI, SFW, SDW, RFW, and RDW. This correlation means that the higher the estimated plant volume, the higher the DQI, and the higher the plant biomass accumulation. Sturdiness quotient was moderately associated with NR and INL.

Table 4. Correlation between morphological characteristics and quality of in vitro raised Melia volkensii plants. The background color indicates strong or weak correlation correlations.

\begin{tabular}{|c|c|c|c|c|c|c|c|c|c|c|c|c|c|c|c|c|c|c|c|}
\hline $\mathrm{PH}$ & 0.62 & & & & & & & & & & & & & & & & & & \\
\hline NL & 0.67 & 0.77 & & & & & & & & & & & & & & & & & \\
\hline NLL & 0.62 & 0.07 & 0.04 & & & & & & & & & & & & & & & & \\
\hline INL & 0.52 & 0.92 & 0.62 & 0.05 & & & & & & & & & & & & & & & \\
\hline CHL & -0.19 & -0.01 & -0.13 & 0.03 & -0.03 & & & & & & & & & & & & & & \\
\hline SD & 0.38 & 0.43 & 0.52 & 0.25 & 0.28 & 0.19 & & & & & & & & & & & & & \\
\hline LA & 0.63 & 0.70 & 0.87 & 0.19 & 0.47 & 0.17 & 0.54 & & & & & & & & & & & & \\
\hline NR & 0.13 & 0.22 & 0.29 & 0.16 & 0.12 & 0.26 & -0.17 & 0.50 & & & & & & & & & & & \\
\hline RL & 0.54 & 0.08 & 0.17 & 0.48 & -0.06 & -0.05 & 0.23 & 0.14 & -0.38 & & & & & & & & & & \\
\hline RD & 0.54 & 0.44 & 0.48 & 0.35 & 0.29 & 0.03 & 0.83 & 0.50 & -0.36 & 0.54 & & & & & & & & & \\
\hline$C D$ & 0.48 & 0.39 & 0.40 & 0.46 & 0.28 & 0.19 & 0.89 & 0.43 & -0.33 & 0.55 & 0.93 & & & & & & & & \\
\hline DQI & 0.44 & 0.34 & 0.34 & 0.36 & 0.19 & 0.12 & 0.77 & 0.41 & -0.32 & 0.51 & 0.96 & 0.89 & & & & & & & \\
\hline SQ & 0.37 & 0.72 & 0.61 & -0.20 & 0.66 & -0.12 & -0.18 & 0.56 & 0.60 & -0.25 & -0.16 & -0.30 & -0.20 & & & & & & \\
\hline RDW & 0.48 & 0.39 & 0.41 & 0.34 & 0.22 & 0.08 & 0.77 & 0.48 & -0.28 & 0.52 & 0.97 & 0.87 & 0.99 & -0.13 & & & & & \\
\hline RFW & 0.44 & 0.46 & 0.50 & 0.26 & 0.29 & 0.18 & 0.83 & 0.57 & -0.21 & 0.44 & 0.96 & 0.89 & 0.97 & -0.08 & 0.98 & & & & \\
\hline SDW & 0.51 & 0.79 & 0.79 & 0.11 & 0.57 & 0.14 & 0.75 & 0.87 & 0.23 & 0.11 & 0.71 & 0.63 & 0.65 & 0.42 & 0.69 & 0.78 & & & \\
\hline SFW & 0.62 & 0.75 & 0.87 & 0.21 & 0.54 & 0.17 & 0.72 & 0.93 & 0.27 & 0.24 & 0.73 & 0.66 & 0.66 & 0.41 & 0.71 & 0.80 & 0.95 & & \\
\hline $\mathrm{S}: \mathrm{R}$ & -0.31 & -0.10 & -0.13 & -0.43 & -0.11 & 0.09 & -0.73 & -0.05 & 0.62 & -0.51 & -0.81 & -0.88 & -0.77 & 0.56 & -0.74 & -0.72 & -0.30 & -0.33 & \\
\hline \multirow[t]{2}{*}{$\mathrm{V}$} & 0.56 & 0.66 & 0.64 & 0.34 & 0.49 & 0.16 & 0.87 & 0.67 & -0.07 & 0.49 & 0.92 & 0.91 & 0.88 & 0.08 & 0.89 & 0.94 & 0.86 & 0.87 & -0.68 \\
\hline & SR & PH & NL & NLL & INL & CHL & SD & LA & NR & RL & RD & $C D$ & DQI & SQ & RDW & RFW & SDW & SFW & S:R \\
\hline
\end{tabular}

SR: survival rate. PH: plant Height (cm). NL: number of leaves per plant. NLL: number of leaflets per leaf. INL: internode length $(\mathrm{mm})$. CHL: chlorophyll (SPAD value). SD: stem diameter $(\mathrm{mm})$. LA: leaf area $\left(\mathrm{cm}^{2}\right)$. NR: number of roots per plant. RL: root length $(\mathrm{cm})$. RD: root diameter $(\mathrm{mm})$. CD: collar diameter $(\mathrm{mm})$. DQI: quality index. SQ: plant height to collar diameter ratio (sturdiness quotient). RFW: root fresh weight (g). RDW: root dry weight (g). SFW: shoot fresh weight (g). SDW: shoot dry weight $(\mathrm{g})$. S:R: shoot to root ratio. V: plant volume. The green color indicates a positive correlation, and the red is negative. The darker the colors, the stronger the correlation, and the lighter the colors, the weaker.

\section{Discussion}

The quality of micropropagated plants after the weaning phase is essential for predicting survival and growth after field planting. In the present study, we assessed the phenotypic variation of a number of selected clones. We used these data to determine the correlations between the observed morphological parameters and calculate quality indices.

\subsection{Survival}

At the end of acclimatization, an average of $85.4 \%$ of the plants survived. The survival rate was genotype-dependent, as significantly more losses were recorded for E34 (field selection) and 19003 (seedling). Survival rate during acclimatization can be a valuable criterion for clonal selection. the variation in the survival rate was similar to that of acclimatizing Persian Walnut genotypes [22]. For Uniola paniculata [23], this variation has been attributed to the genotype-dependent degree of aberrant anatomy of the micropropagated plants. Similar survival rates were previously reported in other Meliaceae families, including M. azedarach L [24], Khaya senegalensis [25], and Toona ciliatae [26]. 


\subsection{Morphological Characteristics of the Shoots}

Significant clonal differences were observed for shoot traits such as plant height, the number of leaves per plant and leaflets, internode length, stem diameter, leaf area, and shoot biomass among clones. This variation among $M$. volkensii clones is attributed to their genetic makeup $[10,27,28]$ and could form an additional basis for further selection. Clone 19016 and 19008 recorded the tallest plants with a long internode compared to other clones; this indicates that they are superior in growth rate. Contradictory results were reported by [29], who observed no significant difference in plant height between Ficus carica L. varieties. Plant height indicates access to sunlight and competition $[30,31]$. Tall plants have more photosynthetic capacity, and they are meant to resist weed competition [32,33].

The greatest number of leaves per plant and maximum leaf area were recorded for 19016. No significant differences were recorded among six clones out of 13, with the number of leaves per plant ranging between 9.57 and 10.27. Four fig tree varieties had a comparable number of leaves during acclimatization [29]. A large number of leaves and leaf areas indicates a high photosynthetic activity. Moreover, leaf area can be used partially for forecasting plant growth [34]. The clones coded MAK1 and 19007 had a larger stem diameter than the others. This can indicate rapid growth and a high survival rate after the outplanting of these clones to semi-arid conditions. Significant differences were observed among clones in chlorophyll (SPAD meter). The chlorophyll of in vitro propagated Prunus africana was reported to increase over time, and the photosynthesis rate was similar to the mother plants [35]. The variation in chlorophyll could be attributed to growth rate and genotypic similarity among clones.

\subsection{Root Traits}

The Melia volkensii seedling produces a thick carrot-like taproot essential for anchoring and storing water and nutrients during the dry period [12], but the roots of the tissuecultured trees branched before thickening. The average number of thick roots ranged from 4.1 to 7.2 , depending on the genotype. The development of multiple taproots could, on the one hand, help plants to survive in semi-arid conditions but, on the other hand, reduce their root depth. This should therefore be determined through multi-year field trials. In Hevea brasiliensis, taproot and lateral root development were attributed to genotypic differences between the clones [36]. A well-established seedling root system facilitates the fast establishment of plants after planting [37]. Root diameter and root length were significantly higher than $1.9 \mathrm{~mm}$ and $8.1 \mathrm{~cm}$, respectively, indicating that the clones were genetically different in developing root traits. A more extensive root system is usually correlated with greater resistance to uprooting under heavy wind conditions [38]. The bigger the diameter of the roots, the more considerable 'carrot' root development. A combination of root length and lateral roots was applicable during the selection of excellent Norway spruce plants [39]. Root growth differences have been reported in Fraxinus pennsylvanica clones [40]. Seedlings with long roots and multiple secondary roots suggested a good water uptake and nutrient use efficiency [41]. Root collar diameter significantly varied with clones, although the minimal collar diameter was slightly above $4 \mathrm{~mm}$ while the maximum was nearly $8 \mathrm{~mm}$. Our results are analogous to those of [42], who described variations in root collar diameter between clones of white spruce. In vitro plants with a larger collar diameter are considered superior because a robust rooting system promises high survival and growth rates after transplantation [32]. Clones significantly differed for root biomass. The heavy root was recorded from MAK1, suggesting that the more mass, the more chances to survive and grow better after planting [32,43].

\subsection{Quality Indexes}

The biomass and quality index of micropropagated M. volkensii clones differed significantly. Clone MAK1, followed by 19016 and 19007, respectively, were the best clones according to their SQ, S:R, plant volume, and DQI. The SQ for all clones was below the threshold value of six. This implies a good expected survival rate in dry areas [18]. The 
results showed that only one of the 13 clones, namely 'MAK 1', had an S:R value less than or equal to two. This confirmed its uniqueness. It indicates that, for MAK1, the shoot is more or less in equilibrium with the root [19]. The SQ and S:R indices were developed for seedlings, and there is little literature on these indices applied to acclimatized in vitro plants.

The plant SQ indicates the vigor and robustness of the nursery tree plants. The clones with the highest robustness had more shoot biomass than roots, suggesting that they may be less resistant to extreme conditions [44], especially in semi-arid environments. After hardening off, transferring in vitro plants to large and long pots can be considered, which may improve the plants' S:R and quality. The planting volume increased in parallel with the increase in the DQI. Seven clones had high DQI values $(\geq 0.2)$. This indicates good quality; the higher this index is, the better the survival and growth after transplanting $[20,43,45]$.

\subsection{Correlations}

Our results suggest that the large, heavy plants with many leaves and leaflets survived the best during the first critical month. Their final NR or RL did not play a role. During acclimatization of Rhus coriaria L., plantlets with high survival rates were longer, but unlike our results, they had more and longer roots [46].

The number of roots negatively correlated with RL, RD, RFW and RDW, CD, and DQI, indicating that clones with more branched roots have shorter and thinner roots. More fibrous roots mean lower quality plants. The negative quality effect of a highly branched main root is not uncommon, as a good M. volkensii seedling is expected to have one deep taproot thickened similar to a carrot [12]. Our results showed that plant height to collar diameter ratio, known as SQ, had a slight negative correlation with SD and RD, root biomass, RL, CHL, and NLL. The plant SQ increased parallelly with PH, NL, INL, NR, and LA. Our findings agree with [47], who reported a significant positive correlation between seedling height and SQ of Castanea sativa Mill. populations.

S:R was negatively correlated with almost all parameters except root length, SQ, and chlorophyll. The high S:R values of evaluated clones indicate an imbalance between the shoot and root system, which negatively influences the quality of the in vitro produced M. volkensii plants. A low shoot-to-root biomass ratio between 1:1 to 1:2 indicates a vigorous plant [18] and a balanced morphological tree that can withstand storms. It also implies that water absorbed by the roots has equilibrium with water lost through transpiration. It also indicates that the amount of water absorbed by the roots is in balance with the transpirational area $[19,48]$. Moreover, DQI and V quality indexes of M. volkensii plants increased progressively with stem and root freshness and dry weight, suggesting that a large biomass may be an indication of high quality. This was similarly found for Acacia spp., Gliricidia sepium, Leucaena spp. [49], and Anthocephalus cadamba [50] whose seedlings' DQI was also associated with biomass.

\section{Conclusions}

The current study showed a significant variation of the phenotypic characteristics and the quality of in vitro produced plantlets between 13 selected $M$. volkensii clones. We demonstrated that, based on indices such as SQ, S:R, V, and DQI, a 'best' clone could be selected (MAK1), followed by 19011, 19016, 19007, KAS5, K.F1, and MAK2, respectively. Correlation analysis revealed that a good quality clone could be recognized by the higher $\mathrm{SD}$, plant biomass, RD, and CD with a low S:R of its derived plants. We assume that these clones will provide insights into mass propagation and genetic improvement in the future. Selection of $M$. volkensii clones based on their quality after hardening off can be a good criterion before mass production. This will help minimize losses after transplanting and during the tree's many years of growth under field conditions.

Author Contributions: Conceptualization, S.P.O.W., G.S., T.M., J.V. and C.D.; methodology, C.D. and S.P.O.W.; investigation, C.D.; resources, S.P.O.W. and G.S.; writing, original draft preparation, C.D. and S.P.O.W.; writing-review and editing, G.S., S.P.O.W., J.M., T.M., F.O. and J.V.; supervision, 
S.P.O.W., J.M. and T.M.; project administration, S.P.O.W. and T.M.; funding acquisition, F.O., G.S., S.P.O.W. and T.M. All authors have read and agreed to the published version of the manuscript.

Funding: This work was supported by Belgian Development Cooperation through the VLIR-UOS team project "Synergy for mass propagation of elite Melia volkensii clones and exploiting its derived biopesticides" Ref KE2018TEA465A103.

Institutional Review Board Statement: Not applicable.

Informed Consent Statement: Not applicable.

Data Availability Statement: The data used to support the findings of this study are included in the main text or in the tables.

Acknowledgments: We greatly acknowledge Kenya Forestry Research Institute (KEFRI) and Better Globe Forestry Ltd. for their assistance.

Conflicts of Interest: The authors declare no conflict of interest.

\section{References}

1. Stewart, M.; Blomley, T. Use of Melia volkensii in a Semi-Arid Agroforestry System in Kenya. Commonw. For. Rev. 1994, 73, 128-131.

2. Mulatya, J.M.; Wilson, J.; Ong, C.; Deans, J.D.; Sprent, J.I. Root Architecture of Provenances, Seedlings and Cuttings of Melia volkensii: Implications for Crop Yield in Dryland Agroforestry. Agrofor. Syst. 2002, 65, 65-72. [CrossRef]

3. Ongerep, S.; Kidia, J.; Masibo, M. Evaluating the Growth Performance of Melia volkensii in Kifu Forest, a Potential Timber Plantation Species in Uganda. Vegetos-Int. J. Plant Res. 2016, 29, 2.

4. $\quad$ Orwa, C.; Mutua, A.; Kindt, R.; Jamnadass, R.; Simons, A. Agroforestree Database: A Tree Reference and Selection Guide Version 4.0: Melia volkensii; World Agroforestry Centre: Nairobi, Kenya, 2009.

5. Kokwaro, O.; John, O. Medicinal Plants of East Africa, 3rd ed.; University of Nairobi Press: Nairobi, Kenya, 2009 ; p. 202.

6. Cantrell, C.L.; Rajab, M.S.; Franzblau, S.G.; Fischer, N.H. Antimycobacterial Triterpenes from Melia volkensii. J. Nat. Prod. 1999, 62, 546-548. [CrossRef]

7. Kamau, R.W.; Juma, B.F.; Baraza, L.D. Antimicrobial Compounds from Root, Stem Bark and Seeds of Melia volkensii. Nat. Prod. Res. 2016, 30, 1984-1987. [CrossRef]

8. Jaoko, V.; Nji, C.; Taning, T.; Backx, S.; Mulatya, J.; Abeele, J.; Olubayo, F.; Mangelinckx, S.; Werbrouck, S.P.O. The Phytochemical Composition of Melia volkensii and Its Potential for Insect Pest Management. Plants 2020, 9, 143. [CrossRef]

9. Jaoko, V.; Taning, C.N.T.; Backx, S.; Motti, P.; Mulatya, J.; Vandenabeele, J.; Magomere, T.; Olubayo, F.; Mangelinckx, S.; Werbrouck, S.P.O.; et al. Laboratory and Greenhouse Evaluation of Melia volkensii Extracts for Potency against African Sweet Potato Weevil, Cylas puncticollis, and Fall Armyworm, Spodoptera frugiperda. Agronomy 2021, 11, 1994. [CrossRef]

10. Runo, M.S.; Muluvi, G.; Odee, D.W. Analysis of Genetic Structure in Melia volkensii (Gurke) Populations Using Random Amplified Polymorphic DNA. Afr. J. Biotechnol. 2004, 3, 421-425. [CrossRef]

11. Werbrouck, S.; Bhogar, N.; Magomere, T.; Omondi, S. In Vitro Biotechnology of Melia volkensii, a High Potential Forestry Tree from Eastern Africa. In Proceedings of the 4th International Union of Forest Research Organizations (IUFRO) Unit 2.09. 02 on Development and Application of Vegetative Propagation Technologies in Plantation Forestry to Cope with a Changing Climate and Environment, Buenos Aires, Argentina, 19-23 September 2016; Volume 4, pp. 249-256.

12. Kenya Forest Service. Guidelines to Growing Melia Volkensii in the Dryland Areas of Kenya; Kenya Forest Service: Nairobi, Kenya, 2018; Volume 53.

13. Luvanda, A.; Musyoki, J.; Takeda, Y. Melia volkensii Enterprises for Enhanced Livelihoods in Semi-Arid Areas in Kenya. In Proceedings of the International Conference on 'Project on Development of Drought Tolerant Trees for Adaptation to Climate Change in Drylands of Kenya', Muguga, Kenya, 13-16 February 2017; Ochieng, D., Kamondo, B., Muturi, G., Eds.; KEFRI: Nairobi, Kenya, 2018; pp. 117-128.

14. Indieka, S.A.; Odee, D.W.; Muluvi, G.M.; Rao, K.N.; Machuka, J. Regeneration of Melia volkensii Gürke (Meliaceae) through Direct Somatic Embryogenesis. New For. 2007, 34, 73-81. [CrossRef]

15. Mulanda, E.S.; Adero, M.O.; Amugune, N.O.; Akunda, E.; Kinyamario, J.I. High-Frequency Regeneration of the Drought-Tolerant Tree Melia volkensii Gurke Using Low-Cost Agrochemical Thidiazuron. Biotechnol. Res. Int. 2012, 2012, 818472. [CrossRef]

16. Murashige, T.; Skoog, F. A Revised Medium for Rapid Growth and Bio Assays with Tobacco Tissue Cultures. Physiol. Plant. 1962, 15, 473-479. [CrossRef]

17. Lloyd, G.; McCown, B. Commercially-Feasible Micropropagation of Mountain Laurel, Kalmia latifolia, by Use of Shoot-Tip Culture. Int. Plant Prop. Soc. Proc. 1980, 30, 421-427.

18. Jaenicke, H. Tree Nursery Practices Practical Guide; World Agroforestry Centre: Nairobi, Kenya, 1999.

19. Haase, D.L. Morphological and Physiological Evaluations of Seedling Quality. In TD National Proceedings: Forest and Conservation Nursery Associations-2006. Proceedings RMRS-P-50. Fort Collins, CO: US Department of Agriculture, Forest Service, Rocky Mountain Research Station; Riley, L., Dumroese, R., Landis, Eds.; USDA: Washington, DC, USA, 2007. 
20. Dickson, A.; Leaf, A.L.; Hosner, J.F. Quality Appraisal of White Spruce and White Pine Seedling Stock in Nurseries. For. Chron. 1960, 36, 10-13. [CrossRef]

21. Nyoka, B.I.; Kamanga, R.; Njoloma, J.; Jamnadass, R.; Mng'omba, S.; Muwanje, S. Quality of Tree Seedlings Produced in Nurseries in Malawi: An Assessment of Morphological Attributes. For. Trees Livelihoods 2018, 27, 103-117. [CrossRef]

22. Yegizbayeva, T.K.; García-García, S.; Yausheva, T.V.; Kairova, M.; Apushev, A.K.; Oleichenko, S.N.; Licea-Moreno, R.J. Unraveling Factors Affecting Micropropagation of Four Persian Walnut Varieties. Agronomy 2021, 11, 1417. [CrossRef]

23. Aracama, C.V.; Kane, M.E.; Wilson, S.B.; Philman, N.L. Comparative Growth, Morphology, and Anatomy of Easy- and Difficultto-Acclimatize Sea Oats (Uniola paniculata) Genotypes during in Vitro Culture and Ex Vitro Acclimatization. J. Am. Soc. Hortic. Sci. 2008, 133, 830-843. [CrossRef]

24. Vila, S.; Scocchi, A.; Mroginski, L. Plant Regeneration from Shoot Apical Meristems of Melia azedarach L. (Meliaceae). Acta Physiol. Plant. 2002, 24, 195-199. [CrossRef]

25. Hung, C.D.; Trueman, S.J. In Vitro Propagation of the African Mahogany Khaya senegalensis. New For. 2011, 42, 117-130. [CrossRef]

26. Li, P.; Shang, Y.; Zhou, W.; Hu, X.; Mao, W.; Li, J.; Li, J.; Chen, X. Development of an Efficient Regeneration System for the Precious and Fast-Growing Timber Tree Toona ciliata. Plant Biotechnol. 2018, 35, 51-58. [CrossRef]

27. Hanaoka, S.; Muturi, G.M.; Watanabe, A. Isolation and Characterization of Microsatellite Markers in Melia volkensii Gurke Conserv. Genet. Resour. 2012, 4, 395-398. [CrossRef]

28. Ndufa, J.K.; Miyashita, H. Preliminary Results on Melia volkensii Clonal Variation in Growth and Wood Properties in the Drylands of Kenya. In Project on Development of Drought Tolerant Trees for Adaptation to Climate Change in Drylands of Kenya; Ochieng, D., Kamondo, B., Muturi, G.M., Eds.; KEFRI: Nairobi, Kenya, 2018; pp. 90-95.

29. Bayoudh, C.; Labidi, R.; Majdoub, A.; Mars, M. In Vitro Propagation of Caprifig and Female Fig Varieties (Ficus carica L.) from Shoot-Tips. J. Agric. Sci. Technol. 2015, 17, 1597-1608.

30. Gavin, D.G.; Peart, D.R. Spatial Structure and Regeneration of Tetramerista glabra in Peat Swamp Rain Forest in Indonesian Borneo. Plant Ecol. 1997, 131, 223-231. [CrossRef]

31. Rijkers, T.; Pons, T.L.; Bongers, F. The Effect of Tree Height and Light Availability on Photosyntheltic Leaf Traits of Four Neotropical Species Differing in Shade Tolerance. Funct. Ecol. 2000, 14, 77-86. [CrossRef]

32. Haase, D.L. Understanding Forest Seedling Quality: Measurements and Interpretation. Tree Plant. Notes 2008, 52, 24-30.

33. Grossnickle, S.C.; MacDonald, J.E. Why Seedlings Grow: Influence of Plant Attributes. Neww For. 2018, 49, 1-34. [CrossRef]

34. Weraduwage, S.M.; Chen, J.; Anozie, F.C.; Morales, A.; Weise, S.E.; Sharkey, T.D. The Relationship between Leaf Area Growth and Biomass Accumulation in Arabidopsis thaliana. Front. Plant Sci. 2015, 6, 167. [CrossRef]

35. Komakech, R.; Kim, Y.G.; Kim, W.J.; Omujal, F.; Yang, S.; Moon, B.C.; Okello, D.; Rahmat, E.; Kyeyune, G.N.; Matsabisa, M.G.; et al. A Micropropagation Protocol for the Endangered Medicinal Tree Prunus africana (Hook f.) Kalkman: Genetic Fidelity and Physiological Parameter Assessment. Front. Plant Sci. 2020, 11, 1871. [CrossRef]

36. Carron, M.P.; Le Roux, Y.; Tison, J.; Dea, B.G.; Caussanel, V.; Clair, J.; Keli, J. Compared Root System Architectures in Seedlings and in Vitro Plantlets of Hevea brasiliensis, in the Initial Years of Growth in the Field. Plant Soil 2000, 223, 73-85. [CrossRef]

37. Davis, A.S.; Jacobs, D.F. Quantifying Root System Quality of Nursery Seedlings and Relationship to Outplanting Performance. New For. 2005, 30, 295-311. [CrossRef]

38. Albrecht, U.; Bodaghi, S.; Meyering, B.; Bowman, K.D. Influence of Rootstock Propagation Method on Traits of Grafted Sweet Orange Trees. HortScience 2020, 55, 729-737. [CrossRef]

39. Högberg, K.A.; Bozhkov, P.V.; Von Arnold, S. Early Selection Improves Clonal Performance and Reduces Intraclonal Variation of Norway Spruce Plants Propagated by Somatic Embryogenesis. Tree Physiol. 2003, 23, 211-216. [CrossRef] [PubMed]

40. Kim, M.S.; Klopfenstein, N.B.; Cregg, B.M. In Vitro and Ex Vitro Rooting of Micropropagated Shoots Using Three Green Ash (Fraxinus pennsylvanica) Clones. New For. 1998, 16, 43-57. [CrossRef]

41. Batista, R.O.; Eduardo, A.; Neto, F.; Fernanda, S.; Deccetti, C.; Viana, C.S. Root Morphology and Nutrient Uptake Kinetics by Australian cedar Clones. Rev. Caatinga 2016, 29, 153-162. [CrossRef]

42. Lamhamedi, M.S.; Chamberland, H.; Bernier, P.Y.; Tremblay, F.M. Clonal Variation in Morphology, Growth, Physiology, Anatomy and Ultrastructure of Container-Grown White Spruce Somatic Plants. Tree Physiol. 2000, 20, 869-880. [CrossRef] [PubMed]

43. Tsakaldimi, M.; Ganatsas, P.; Jacobs, D.F. Prediction of Planted Seedling Survival of Five Mediterranean Species Based on Initial Seedling Morphology. New For. 2013, 44, 327-339. [CrossRef]

44. Takoutsing, B.; Tchoundjeu, Z.; Degrande, A.; Asaah, E.; Gyau, A.; Nkeumoe, F.; Tsobeng, A. Assessing the Quality of Seedlings in Small-Scale Nurseries in the Highlands of Cameroon: The Use of Growth Characteristics and Quality Thresholds as Indicators. Small-Scale For. 2014, 13, 65-77. [CrossRef]

45. Dina, K.D.; Pande, T.; Vladimir, M.; Mare, T. Dynamic of Growth and Quality of Arizona Cypress (Cupressus arizonica Greene) Seedlings from Three Container Types. In Proceedings of the International Conference Reforestation Challenges, Belgrade, Serbia, 3-6 June 2015. [CrossRef]

46. Amiri, S.; Mohammadi, R. Establishment of an Efficient in Vitro Propagation Protocol for Sumac (Rhus coriaria L.) and Confirmation of the Genetic Homogeneity. Sci. Rep. 2021, 11, 173. [CrossRef]

47. Tumpa, K.; Vidaković, A.; Drvodelić, D.; Šango, M.; Idžojtić, M.; Perković, I.; Poljak, I. The Effect of Seed Size on Germination and Seedling Growth in Sweet Chestnut (Castanea sativa Mill.). Forests 2021, 12, 858. [CrossRef] 
48. Bernier, P.Y.; Lamhamedi, M.S.; Simpson, D.G. Shoot: Root Ratio Is of Limited Use in Evaluating the Quality of Container Conifer Stock. Tree Plant. Notes 1995, 46, 102-106.

49. Bayala, J.; Dianda, M.; Wilson, J.; Ouédraogo, S.J.; Sanon, K. Predicting Field Performance of Five Irrigated Tree Species Using Seedling Quality Assessment in Burkina Faso, West Africa. New For. 2009, 38, 309-322. [CrossRef]

50. Budiman, B.; Sudrajat, D.J.; Lee, D.K.; Kim, Y.S. Effect of Initial Morphology on Field Performance in White Jabon Seedlings at Bogor, Indonesia. For. Sci. Technol. 2015, 11, 206-211. [CrossRef] 